\title{
Beta-Dependent Upper Bound On Ion Temperature Anisotropy In A Laboratory Plasma
}

Paul Keiter

Earl Scime

Matthew Balkey

Robert Boivin

John Kline

See next page for additional authors

Follow this and additional works at: https://researchrepository.wvu.edu/faculty_publications

\section{Digital Commons Citation}

Keiter, Paul; Scime, Earl; Balkey, Matthew; Boivin, Robert; Kline, John; and Gary, S, "Beta-Dependent Upper Bound On Ion Temperature Anisotropy In A Laboratory Plasma" (2000). Faculty Scholarship. 476.

https://researchrepository.wvu.edu/faculty_publications/476 


\section{Authors}

Paul Keiter, Earl Scime, Matthew Balkey, Robert Boivin, John Kline, and S Gary 


\title{
LETTERS
}

The purpose of this Letters section is to provide rapid dissemination of important new results in the fields regularly covered by Physics of Plasmas. Results of extended research should not be presented as a series of letters in place of comprehensive articles. Letters cannot exceed four printed pages in length, including space allowed for title, figures, tables, references and an abstract limited to about 100 words. There is a three-month time limit, from date of receipt to acceptance, for processing Letter manuscripts. Authors must also submit a brief statement justifying rapid publication in the Letters section.

\section{Beta-dependent upper bound on ion temperature anisotropy in a laboratory plasma}

\author{
Paul A. Keiter, Earl E. Scime, Matthew M. Balkey, Robert Boivin, and John L. Kline \\ West Virginia University, Morgantown, West Virginia 26506 \\ S. Peter Gary \\ Los Alamos National Laboratory, Los Alamos, New Mexico 87544
}

(Received 25 August 1999; accepted 9 November 1999)

\begin{abstract}
Laser induced fluorescence measurements of ion temperatures, parallel and perpendicular to the local magnetic field, in the Large Experiment on Instabilities and Anisotropies space simulation chamber (a steady-state, high beta, argon plasma) display an inverse correlation between the upper bound on the ion temperature anisotropy and the parallel ion beta $\left(\beta=8 \pi n k T / B^{2}\right)$. These observations are consistent with in situ spacecraft measurements in the Earth's magnetosheath and with a theoretical/computational model that predicts that such an upper bound is imposed by scattering from enhanced fluctuations due to growth of the ion cyclotron anisotropy instability (the Alfvén ion cyclotron instability). (C) 2000 American Institute of Physics.
\end{abstract}

[S1070-664X(00)05102-8]

In a carefully controlled, fully diagnosed, high beta, plasma, we have carried out the first laboratory demonstration of the existence of an upper bound on the ion temperature anisotropy that scales inversely with the plasma beta $\left(\beta=8 \pi n k T / B^{2}\right)$. This experiment confirms the predictions of collisionless theory and simulations, as well as validating results of space plasma observations carried out with less comprehensive diagnostics and under natural, rather than controlled conditions.

If the ion velocity distribution is approximately biMaxwellian and $T_{i \perp}>T_{i \|}$, where perpendicular and parallel denote directions relative to the background magnetic field $B_{0}$, then two distinct modes may grow. These are the electromagnetic ion cyclotron anisotropy instability (also known as the Alfvén Ion Cyclotron instability) ${ }^{1-3}$ and the mirror instability. ${ }^{4,5}$ The former has a real frequency $\omega_{r}$ which satisfies $0<\omega_{r}<\Omega_{i}\left(\Omega_{i}\right.$ is the ion cyclotron frequency), whereas $\omega_{r}=0$ for the mirror mode in a homogeneous plasma. The magnetic fluctuations of the ion cyclotron anisotropy mode are directed primarily perpendicular to $B_{0}$. Mirror mode fluctuations are primarily compressive, that is, $\Delta B \| B_{0}$. The threshold anisotropy for the ion cyclotron anisotropy mode is a function of the ionic constituents of the plasma $^{6}$ and typically has the lower threshold when $\beta_{i \|}$ $=8 \pi n k T_{i \|} / B_{0}^{2}<1$. The mirror instability is more likely to arise under conditions of higher parallel ion beta. ${ }^{7}$

Linear Vlasov theory indicates that the threshold condition for onset of the ion cyclotron anisotropy instability for a fixed value of the dimensionless maximum growth rate $\gamma / \Omega_{i}$ in an electron/ion plasma can be written as ${ }^{8}$

$$
\left(T_{i \perp} / T_{i \|}\right)-1=S_{p} / \beta_{i \|^{p}}^{\alpha} .
$$

Here $S_{p}$ and $\alpha_{\mathrm{p}}$ are fitting parameters. $S_{p}$ is a dimensionless number of the order of unity and is determined by the choice of maximum growth rate. $\alpha_{p}$ is relatively independent of $\gamma / \Omega_{i}$ with typical values of $\alpha_{p} \approx 0.4$.

There is a long-standing general hypothesis that waveparticle scattering by enhanced fluctuations from kinetic plasma instabilities should constrain the anisotropies that drive the unstable modes. A corollary, apparently first advocated by Manheimer and Boris, ${ }^{9}$ is that a plasma instability threshold derived from linear theory should correspond to an observable bound on the anisotropy driving the unstable mode. Recent hybrid simulations of ion temperature anisotropy instabilities ${ }^{7}$ have provided support for this corollary.

However, it is difficult to test this hypothesis and its consequences with space plasma data because particle distributions are often observed to exhibit more than one type of non-Maxwellian feature and scattering may be due to more than one microinstability. In some cases, the Earth's bow shock provides enough heating to the solar wind ions in the direction perpendicular to $B_{0}$ that $T_{i \perp}>T_{i \|}$ is the predominant proton free energy. The magnetic field becomes compressed as the magnetosheath plasma flows toward the dayside magnetopause and $\beta_{i \|}$ decreases. Under these conditions, a number of observers ${ }^{10-13}$ have demonstrated 


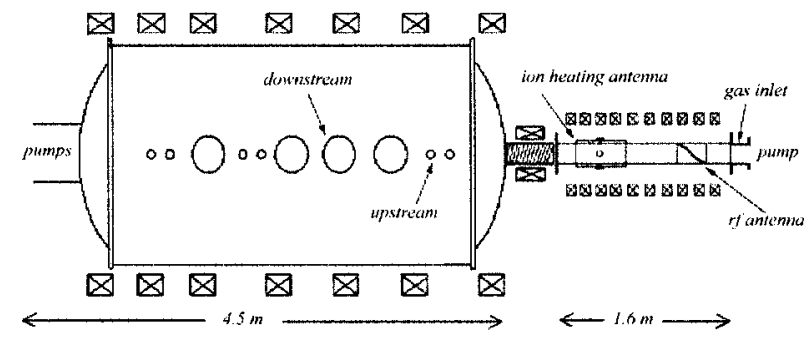

FIG. 1. The Large Experiment on Instabilities and Anisotropies (LEIA) connected to the helicon plasma source (right). The two locations at which LIF measurements were performed in LEIA are indicated.

that the proton temperature anisotropy for $\beta_{i \|}<1$ does indeed have an upper bound of the form of Eq. (1) with 0.4 $<\alpha_{p}<0.6$. Several of these observations include measurements of proton-cyclotron-like fluctuations. ${ }^{1}$

The ion cyclotron anisotropy instability has been examined before in laboratory plasmas, ${ }^{14}$ most recently in the GAMMA 10 mirror experiment. ${ }^{15}$ Although the GAMMA 10 results did demonstrate a correlation between $T_{i \perp} / T_{i \|}$, $\beta_{i \perp}$, and the presence of Alfvén ion cyclotron instability waves, they did not indicate the existence of an upper bound on the ion temperature anisotropy that is inversely correlated with $\beta_{i||}$. In fact, the reported results show no evidence of an upper bound on the ion temperature anisotropy even though the ion temperature anisotropy exceeded the threshold for instability excitation. ${ }^{16}$ Thus, there are significant differences between the previous laboratory experiments, the magnetosheath observations, and the linear Vlasov theory results.

In this paper, we present our direct measurements of the perpendicular and parallel ion distribution functions in a homogeneous, marginally collisional plasma and our observations of an inverse correlation between the ion temperature anisotropy upper bound and $\beta_{i \|}$. We also present measurements of the spectrum of transverse, electromagnetic fluctuations and show that, as would be expected for the ion cyclotron anisotropy instability, there is a correlation between thermalization of the ion distribution and sub-cyclotron frequency fluctuations.

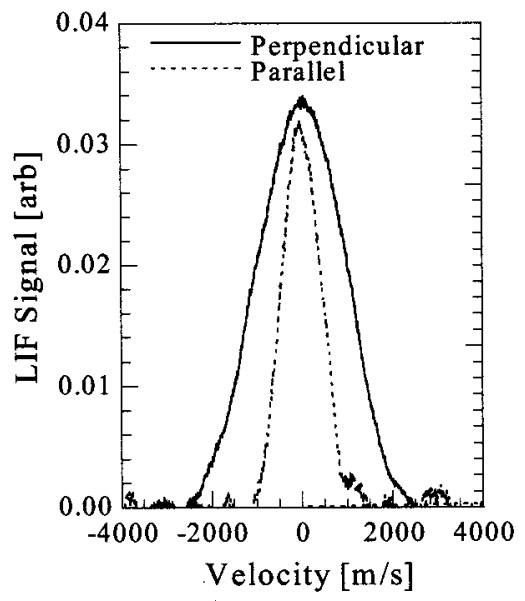

FIG. 2. The parallel and perpendicular ion velocity space distributions functions in LEIA measured with LIF at the upstream position. For this data, $T_{i \perp}$ $=0.37 \mathrm{eV}$ and $T_{i \|}=0.07 \mathrm{eV}$. The parallel data has been artificially scaled to an amplitude comparable to the perpendicular data.

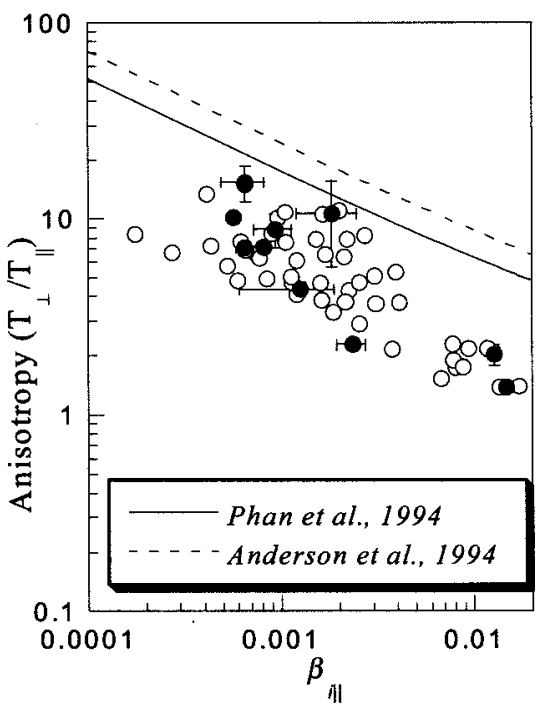

FIG. 3. The ion temperature anisotropy, $A=T_{i \perp} / T_{i \|}$, versus $\beta_{i \|}$ (open circles) measured at the upstream position. These data were obtained over a wide range of operating magnetic fields but at fixed if power and neutral pressure. Also shown are averaged values of anisotropy and for similar operating conditions (solid circles) investigated on different days with standard deviation error bars and extrapolations of the fits from the magnetospheric observations (Refs. 11 and 12).

The experiments were performed in the Large Experiment on Anisotropies and Instabilities (LEIA). LEIA (Fig. 1) consists of a steady state, high density, helicon plasma source coupled to a large ( $4.4 \mathrm{~m}$ long, $1.8 \mathrm{~m}$ inner diameter) vacuum chamber. Typical LEIA argon plasma parameters for these experiments were $n \leqslant 10^{12} \mathrm{~cm}^{-3}, B \approx 17-70 \mathrm{G}$, $T_{e} \approx 4-10 \mathrm{eV}, T_{i} \approx 0.1-1.0 \mathrm{eV}, \beta_{e \|} \leqslant 0.2$ and $\beta_{i \|} \leqslant 0.02$. Because of the differential pumping scheme, the neutral pressure decreases from 1.4 mTorr in the source where the gas is injected to 0.2 mTorr in LEIA. At these ion temperatures and magnetic fields, the plasma diameter is 16 times the ion gyroradius. The plasma is marginally collisional as $\lambda_{m f p} / \rho \approx 1$ for the ion-ion collisions $\left(\lambda_{m f p}\right.$ is the mean free path and $\rho$ is the ion gyroradius) and based on the edge neutral pressure $\lambda_{m f p} / \rho \approx 1$ for ion-neutral collisions. ${ }^{17}$ This is an overestimate for ion-neutral collisions as the neutral pumping effect in helicon plasmas should lead to a significantly reduced neutral pressure on axis in LEIA. ${ }^{18}$ The ionion collision frequency ranges from 5 to 10 times the ion gyrofrequency.

The parallel and perpendicular ion temperatures in LEIA were determined by laser induced fluorescence (LIF). The ion velocity distribution is bi-Maxwellian with $T_{i \perp}>T_{i \|}$ (Fig. 2). Previous LIF measurements have demonstrated that the perpendicular ion temperature is many times the parallel ion temperature in the helicon source and that the level of ion temperature anisotropy can be controlled by adjusting the source magnetic field ${ }^{19}$ or by auxiliary ion-heating. ${ }^{20}$

The ion temperature anisotropy, $T_{i \perp} / T_{i \|}$, versus $\beta_{i \|}$ in LEIA is shown in Fig. 3. The data shown in Fig. 3 were all obtained for the same neutral pressure and source RF power. Different source magnetic fields, LEIA magnetic fields, and auxiliary ion heating power levels $(0$ or $200 \mathrm{~W})$ were used to vary the anisotropy and $\beta_{i \|}$. Also shown in Fig. 3 are the fits 

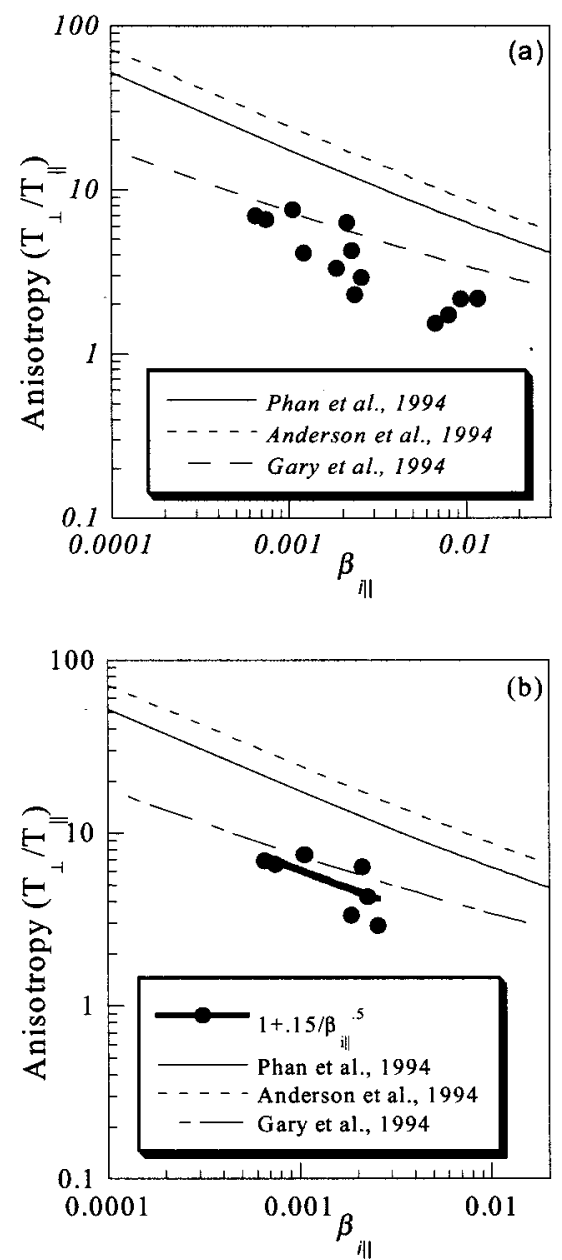

FIG. 4. (a) A subset of the ion temperature anisotropy, $A=T_{i \perp} / T_{i \|}$, versus data of Fig. 3 selected so that the isotropization rate, Eq. (2), varies by less than $11 \%$. (b) The same data of Fig. 4(a) with the additional constraint of fixed ion-neutral collision frequency (less than $11 \%$ variation). Also shown are the fits to Eq. (1) for the laboratory measurements, the magnetospheric observations (Refs. 11 and 12) and the linear Vlasov calculations with $\gamma$ $=10^{-4} \Omega_{p}{ }^{7}$

to the two-dimensional magnetospheric ion measurements of Anderson et al. $\left(S_{p}=0.85, \alpha_{p}=0.48\right)^{13}$ and the threedimensional measurements of Phan et al. $\left(S_{p}=0.63, \alpha_{p}\right.$ $=0.50){ }^{12}$

The linear Vlasov model assumes a collisionless plasma and that wave-particle interactions provide the anisotropy limiting velocity space diffusion typically provided by Coulomb collisions in a collisional plasma. Because these experiments were performed in a marginally collisional plasma, it is important to determine if collisions are responsible for the observed scaling of anisotropy with $\beta_{i \|}$. Ignoring the much smaller velocity space diffusion arising from ion-electron collisions, the rates of change for the perpendicular and parallel ion temperatures $\operatorname{are}^{21}$

$$
d T_{\perp} / d t=-\frac{1}{2} d T_{\|} / d t=-\nu\left(T_{\perp}-T_{\|}\right),
$$

where

$$
\nu \approx \frac{2 \sqrt{\pi} e^{4} n}{\sqrt{m_{i}}\left(k T_{\|}\right)^{3 / 2} A^{2}}\left[-3+(A+3) \frac{\tan ^{-1}(\sqrt{A})}{\sqrt{A}}\right]
$$

and $A=T_{i \perp} / T_{\|}$. For typical LEIA parameters,

$$
\nu \approx 4 \sqrt{\pi} e^{4} n / \sqrt{m_{i}}\left(k T_{\|}\right)^{3 / 2} A^{0.9} .
$$

Rewriting $\beta_{i \|}$ in terms of Eq. (4),

$$
\beta_{i \|} \approx \nu\left(k T_{\|}\right)^{5 / 2} A^{0.9} / 4 \sqrt{\pi m_{i}} e^{4} B^{2} .
$$

Thus, varying the magnetic field and the ion temperatures can yield a wide range of $\beta_{i||}$ for a fixed isotropization frequency, $\nu$, due to ion-ion collisions.

Figure 4(a) shows a subset of the data shown in Fig. 3. For these data, the isotropization frequency described by Eq. (3) varies by less than $11 \%$. Note that $\beta_{i \|}$ spans more than order of magnitude and the temperature anisotropy changes by a factor of 3 . In addition to ion-ion collisions, ion-neutral collisions can also reduce temperature anisotropy through velocity space diffusion. Assuming a constant cross section (a reasonable approximation over the limited energy range of interest), the ion-neutral collision frequency is proportional to $\sqrt{T_{i}}$, where $T_{i}^{2}=\left(2 T_{i \perp}^{2}+T_{i \|}^{2}\right) / 3$. Figure 4(b) shows a subset of the data shown in Fig. 4(a). For these data, both the isotropization and ion-neutral collision frequencies vary by less than $11 \%$. Although the range of $\beta_{i \|}$ is reduced, the inverse correlation of ion temperature anisotropy with $\beta_{i \|}$ apparent in Fig. 3 is still apparent. A fit of Eq. (1) to the data yields $S_{p}=0.15$ and $\alpha_{p}=0.5$. The fact that the full data set shown in Fig. 3 falls off more steeply at higher $\beta_{i \|}$ is likely due to the increasingly more important effects of ion-ion collisions at larger values of plasma density which result in larger values of $\beta_{i \|}$.

Also shown in Fig. 4 is a linear Vlasov theory curve for the onset of the ion cyclotron anisotropy instability at a maximum growth rate of $\gamma / \Omega_{i}=10^{-4}$. The scaling of the laboratory data with parallel ion beta, $\alpha_{p} \sim 0.5$, is consistent with both the spacecraft measurements and the theory. In a serendipitous way, this laboratory experiment reproduces the evolution of ion distributions in the terrestrial magnetosheath. At some distant location, a temperature anisotropy is created. The plasma is then examined at a different spatial location in the magnetosheath and the temperature anisotropy is observed to be limited in a way that depends on the plasma parameters at the point of measurement. The linear Vlasov models suggest that overall magnitude of the anisotropy, the $S_{p}$ term in Eq. (1), also depends on the level of "driving" or initial anisotropy. ${ }^{8}$ Numerically, this is varied by choosing different maximum growth rates in the simulation. The maximum growth rate that best fits the laboratory data is clearly smaller than what is required to match the spacecraft measurements.

Computations of the amplitude of the electromagnetic fluctuations needed to limit the ion temperature anisotropy are on the order of $1 \%$ of the background magnetic field for moderate initial anisotropies and $\beta_{i \|} \approx 0.1{ }^{7}$ For Alfvén ion cyclotron waves, the fluctuations should be transverse, right circularly polarized waves at frequencies below the ion cyclotron frequency. Electromagnetic wave measurements in LEIA (Fig. 5) were obtained with an array of threedimensional magnetic sense coils. ${ }^{22}$ Scans of helicon source and LEIA parameters clearly indicate that the amplitude of the sub-cyclotron waves depends strongly on the ion beta in 


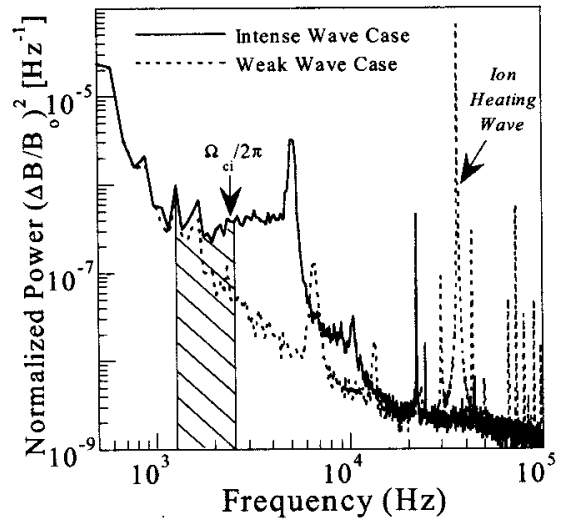

FIG. 5. Transverse electromagnetic wave activity in LEIA. Strong wave activity case (solid line), weak wave activity case (dashed line). The weak wave activity case is comparable to the background level of the probe. After background subtraction, the integrated field perturbation over $0.5 \Omega_{i}$ to $1 \Omega_{I}$ is roughly $1 \%$ of the background magnetic field. The dashed lines mark the region of integration.

LEIA, while the higher frequency peaks (around $6 \mathrm{kHz}$ in Fig. 5) depend strongly on source parameters. Preliminary measurements suggest that the higher frequency waves may play a role in creating the ion temperature anisotropy in the expanding field region between LEIA and the source. Note that the ion cyclotron frequency identified in Fig. 5 is based on the magnetic field strength at the magnetic probe. Towards the source, the magnetic field increases sharply. AIC waves excited upstream at a fraction of the upstream cyclotron frequency could appear at a larger fraction of the cyclotron frequency at the probe location, i.e., waves excited at $\omega \sim 0.25 \Omega_{I}$ upstream might appear at $\omega \sim 0.75 \Omega_{I}$ downstream. For AIC waves, however, waves generated upstream at frequencies above the local ion cyclotron frequency should not reach the probe.

Near the ion cyclotron frequency, the transverse wave amplitude $\left(B_{r}\right)$, is more than 10 times the parallel, compressional $\left(B_{z}\right)$, wave amplitude. After background subtraction, integration of the measured transverse fluctuation wave amplitude from $0.5 \Omega_{i}$ to $1 \Omega_{i}$ yields a sub-cyclotron fluctuation amplitude between 0.5 to $1 \%$ of the background magnetic field. Additional simulations are needed to determine if the larger driving anisotropies in this $\beta_{i \|} \approx 0.001$ experimental case can explain magnetic fluctuation amplitudes on the order of $1 \%$.

To investigate the correlation of the sub-cyclotronic wave activity with relaxation of the ion temperature anisotropy, LIF measurements were performed at two axial locations in LEIA (shown in Fig. 1). While keeping the isotropization and ion-neutral collision frequencies as constant as possible, the RF and ion heating powers were manipulated to vary the upstream ion temperature anisotropy and amplitude of the sub-cyclotronic wave activity. The neutral pressure, helicon source magnetic field, and LEIA magnetic field were held constant. When the ion heating circuit was engaged, the sub-cyclotronic wave power decreased from roughly $1 \%$ of the background field (the strong wave activity case shown in Fig. 5) to the noise level (the weak wave activity case shown in Fig. 5). The physics behind the suppression of the low frequency wave activity is not understood at this time. The ratio of upstream anisotropy to downstream anisotropy decreased from $A_{\text {up }} / A_{\text {down }}=19 / 2=9.5$ in the case of strong wave activity and from $A_{\text {up }} / A_{\text {down }}=17 / 3=5.7$ in the case of weak wave activity. Thus, stronger sub-cyclotronic wave activity appears to be correlated with more effective thermalization of the ion distribution, i.e., a larger change in the upstream and downstream anisotropies.

This work demonstrates that Eq. (1) with $\alpha_{p} \approx 0.5$ constitutes an observable constraint on $T_{i \perp} / T_{i \|}-1$ and that the observed sub-cyclotron magnetic field fluctuations are correlated with isotropization of the ions. The observed fluctuations are electromagnetic, transverse, and the measured wave numbers ${ }^{22}$ are consistent with predictions for ion cyclotron anisotropy waves. These results suggest that the ion cyclotron anisotropy instability is indeed the process that imposes this bound in both magnetosheath and high $\beta_{i \|}$ laboratory plasmas. These results also suggest that this constraint is a fundamental property of all high beta plasmas bearing such an anisotropy, including other laboratory plasmas as well as space and astrophysical domains not yet subject to in situ observations. Thus, the basic concept of short wavelength instabilities imposing anisotropy constraints offers an alternative to the long-standing approach of using analogues of collision-dominated transport coefficients to express the consequences of small-scale, collective processes in the collisionless plasmas of space. For example, the Manheimer and Boris limitation on field-aligned currents ${ }^{9}$ may prove more useful than the various approaches to anomalous resistivity, ${ }^{23}$ and the heat flux constraint imposed by heat flux instabilities may have greater application than the Spitzer-Harm thermal conductivity derived from collision-dominated theory. ${ }^{24}$

\section{ACKNOWLEDGMENTS}

This work supported by NSF under Grant No. ATM9616467 and the U.S. Department of Energy under Grant No. DE-FG02-97ER54420.

${ }^{1}$ C. F. Kennel and H. E. Petschek, J. Geophys. Res. 71, 1 (1966).

${ }^{2}$ R. C. Davidson and Joan M. Ogden, Phys. Fluids 18, 1045 (1975).

${ }^{3}$ Gary R. Smith, Phys. Fluids 27, 1499 (1984).

${ }^{4}$ S. Chandrasekhar, N. Kaufman, and K. M. Watson, Proc. R. Soc. London, Ser. A 245, 435 (1958).

${ }^{5}$ A. Barnes, Phys. Fluids 9, 1483 (1966).

${ }^{6}$ C. P. Price, D. W. Swift, and L.-C. Lee, J. Geophys. Res. 91, 101 (1986).

${ }^{7}$ S. P. Gary, J. Wang, D. Winske, and S. A. Fuselier, J. Geophys. Res. 102, 27159 (1997).

${ }^{8}$ S. P. Gary, M. E. McKean, D. Winske, B. J. Anderson, R. E. Denton, and S. A. Fuselier, J. Geophys. Res. 99, 5903 (1994).

${ }^{9}$ W. Manheimer and J. P. Boris, Comments Plasma Phys. Control. Fusion 3, 15 (1977).

${ }^{10}$ B. J. Anderson and S. A. Fuselier, J. Geophys. Res. 98, 1461 (1993).

${ }^{11}$ T.-D. Phan, G. Paschmann, W. Baumjohann, and N. Sckopke, J. Geophys. Res. 95, 1015 (1994).

${ }^{12}$ B. J. Anderson, S. A. Fuselier, S. P. Gary, and R. E. Denton, J. Geophys. Res. 99, 5877 (1994).

${ }^{13}$ L. C. Tan, S. F. Fung, R. L. Kessel, S. H. Chen, J. L. Green, and T. E. Eastman, Geophys. Res. Lett. 25, 587 (1998).

${ }^{14}$ T. A. Casper and Gary R. Smith, Phys. Rev. Lett. 48, 1015 (1982).

${ }^{15}$ R. Katsumata, M. Ichimura, M. Inutake, H. Hojo, A. Mase, and T. Tamano, Phys. Plasmas 3, 4489 (1996). 
${ }^{16}$ M. Ichimura, M. Inutake, R. Katsumata, N. Hino, H. Hojo, K. Ishii, T. Tamano, and S. Miyoshi, Phys. Rev. Lett. 70, 2734 (1993).

${ }^{17}$ V. E. Golant, A. P. Zhilinsky, and I. E. Sakharov, Fundamentals of Plasma Physics (Wiley, New York, 1977), pp. 51.

${ }^{18}$ J. Gilland, R. Bruen, and N. Hershkowitz, Plasma Sources Sci. Technol. 7, 416 (1998).

${ }^{19}$ E. E. Scime, P. A. Keiter, M. W. Zintl, M. M. Balkey, J. L. Kline, and M. E. Koepke, Plasma Sources Sci. Technol. 7, 186 (1998).
${ }^{20}$ J. L. Kline, E. E. Scime, P. A. Keiter, M. M. Balkey, and R. F. Boivin, Phys. Plasmas (in press).

${ }^{21}$ J. D. Huba, NRL Plasma Formulary (Naval Research Laboratory, Washington, DC, 1994), p. 33.

${ }^{22}$ P. A. Keiter, Ph.D. thesis, West Virginia University, 1999.

${ }^{23}$ F. V. Coroniti, Space Sci. Rev. 42, 399 (1985).

${ }^{24}$ E. E. Scime, S. Gary, J. L. Phillips, and W. C. Feldman, J. Geophys. Res. 99, 23391 (1994). 